\title{
Surface Films on Poly(oxymethylene) Single Crystals*
}

\author{
Janice Breedon Jones and P. H. Geil**
}

(June 30, 1975)

\begin{abstract}
The deformation of single crystals of poly(oxymethylene) grown from 0.01 percent bromobenzene solution has been studied by deposition on a deformable substrate. Slight decoration of the crystal surfaces with gold prior to mechanical deformation of the composite reveals breaks in the gold which are displaced with respect to cracks in the underlying polymer crystals. These observations are interpreted to imply the existence of a very thin discrete film on the surface of the polymer crystals which can slip durino deformation. Such a film might arise from polymer molecules adsorbed on the crystal surface.
\end{abstract}

Key words: Adsorbed polymer, deformation, fold surface, gold decoration, polymer crystals, poly(oxymethylene), surface layer.

In the course of some investigations $[1]^{1}$ on the formation of microcracks in single crystals of poly(oxymethylene) (POM), some intriguing observations have been made which appear to shed some light on the nature of the fold surfaces of solution-grown crystals.

\footnotetext{
*An invited paper

**Present address: Case Western Reserve University, Department of Macromolecula Science, Cleveland, Ohio 44106

'Figures in brackets indicate the literature references at the end of this paper.
}

The crystals were of Delrin 500 grown by ambient cooling from 0.01 percent solution in bromobenzene. They were deposited on Mylar film to be stretched using the method first developed by Geil [2], and were lightly decorated with gold in a diffusion pump vacuum before being drawn. The crystals were stretched in air at room temperature or at $96{ }^{\circ} \mathrm{C}$.

Figure 1 is an electron micrograph of some crystals which were gold decorated, drawn at room temperature,

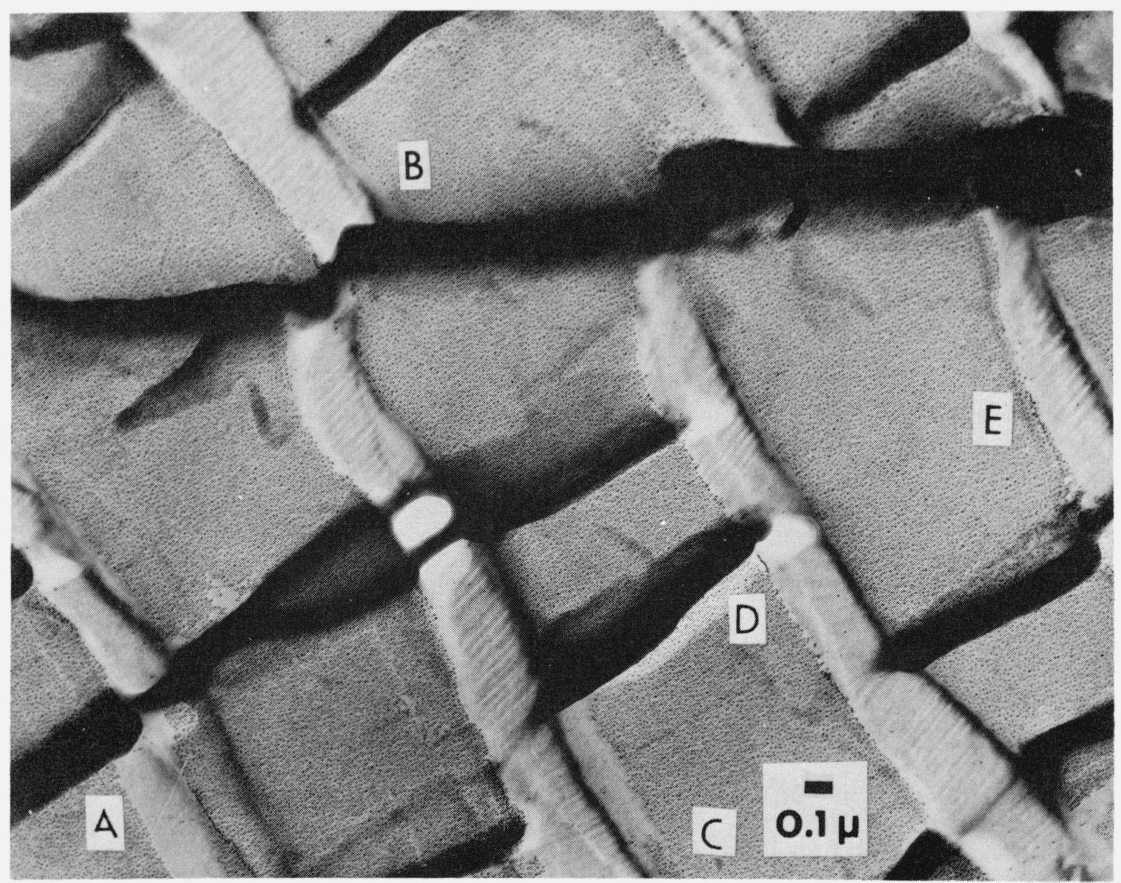

FIGURE 1. POM single crystal gold decorated before draw and shadowed obliquely with $\mathrm{Pt} / \mathrm{C}$ parallel to the draw direction after it was drawn. 
and then shadowed obliquely with platinum/carbon to reveal the topography of the drawn crystal. In spite of the existence of well-separated gold particles on the surface, the gold has deformed as a separate layer. Only a few of the gold particles have been pulled out onto the fibrils bridging the cracks. The gold "layer" seems to have broken in a brittle manner at the microcracks, but the break in the gold is often displaced with respect to the actual crack. This is particularly conspicuous at the points marked with the letters. At point A, the gold layer is completely displaced from the crack, possibly even sagging into it. At point $\mathrm{B}$, the gold appears to be hanging over the edge on one side of the crack, while not reaching the edge on the other side. At point C, a narrow strip of the gold layer has been bent off the main mass as the two edges separated. Estimation of the thickness of the layer supporting the gold was not possible. Where a shadow could be seen, it appeared to be cast mostly by the gold particles and was certainly several times shorter than that cast by single lamellae of spiral growths seen in other micrographs of the same preparation. Therefore, it is highly unlikely that the layer is simply an upper crystal lamella that is being displaced across the lower crystals.

Figure 2 is an electron micrograph of some crystals that were gold decorated and then stretched at $96{ }^{\circ} \mathrm{C}$. Again the breaks in the gold are displaced with respect to the cracks, and the displacements are not all in the same direction. The breaks in the gold corresponding to cracks $\mathrm{A}$ and $\mathrm{B}$ are shifted toward each other. Also, cracks $\mathrm{B}$ and $\mathrm{C}$, whose tips meet at a pleat, show opposite displacement of the gold. Also visible in the cracks (and to a lesser extent in those in fig. 1) is a diffuse darkening of the edges and fibrils which appears to be decoration by residual poly(acrylic acid) used in the preparation of the samples [1].

While not all cracks in all crystals showed this displacement of the gold particles, it was seen often enough to be a phenomenon that appears to be typical of these crystals. The evidence points to the existence of a discrete film on the fold surface which apparently can slip across it during deformation. The film may even be somewhat elastic, as suggested by the region in figure 2 where the film seems either to have shrunk or not to have stretched enough between two neighboring cracks. In other micrographs, regions could be seen in which some of the gold at both ends of a given fibril had been pulled noticeably toward, but never onto, the fibril. These observations all suggest that the film is flexible and coherent with a small amount of ductility.

The most likely explanation for this surface film is that it consists of adsorbed polymer molecules. This would account for the observed flexibility, coherence, and possible elasticity. That it might be cilia extending from the crystal seems unlikely because of the extent of the displacement and the fact that the displacement is always in the same direction on both sides of a crack. The possibility exists that the layer is some type of contamination introduced before or during the gold evaporation step. However, contamination such as pump oil from the vacuum system [3] would probably have such low molecular weight that it would either lack the coherence necessary for the effects seen in the micrographs.

Observations of an approximately $200 \AA$ wide region at the edges of similar cracks in Penton and poly (4-

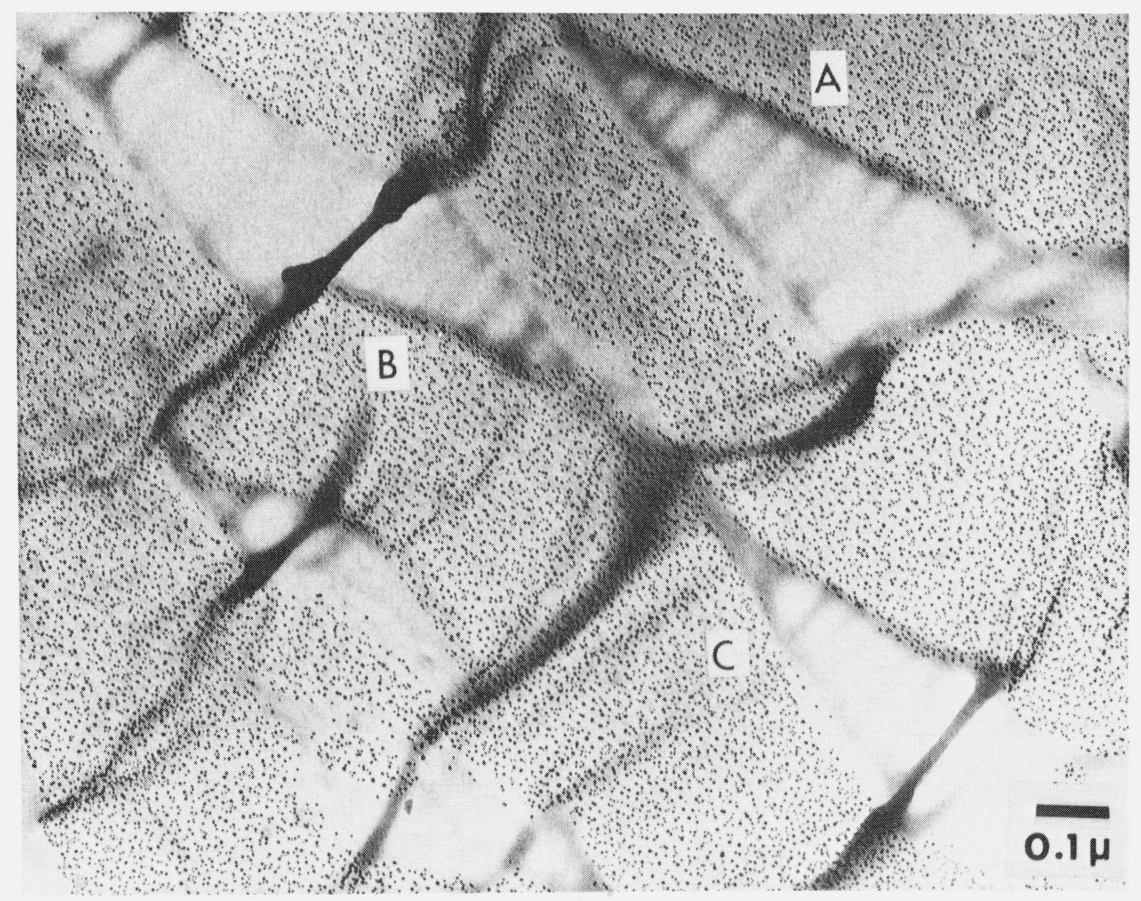

Figure 2. POM single crystal gold decorated before draw at $96{ }^{\circ} \mathrm{C}$. 
methyl pentene-1) crystals, which region has surface properties differing from the rest of the crystal [1], may be due to a surface film similar to that found for POM. In these preparations, the crystal appears to have been drawn out from underneath the film, thus exposing the fold surface.

Such a polymer film has been postulated by Lauritzen and Hoffman [4] and Hoffman, Davis, and Lauritzen [5] as part of some recent work on the theory of polymer crystallization. They suggest that before molecules are added onto the growth face of the crystal, they are first adsorbed onto the lateral and/or fold surfaces of the growing crystal, and then migrate across the surface to the growth sites. A film, possibly in equilibrium with the solution, of adsorbed molecules that never reached the growth face would likely be left on the surface of the crystal. The film could also be chains that for some reason were rejected from the growing crystal, or adsorbed chains that play no part in the crystallization process. Also, the possibility cannot yet be completely ruled out that polymer molecules were deposited onto the fold surface (and Mylar) while the crystal suspension was drying on the Mylar before deformation. An attempt was made to find such a film at cracks in a thin layer of brittle carbon that had been evaporated onto some Mylar before crystals were deposited and drawn. The carbon formed large cracks across which both the crystals and any film between them from the deposition process would be drawn. However, no slippage of the gold layer relative to the cracks was seen in the regions between the crystals, suggesting that the film only exists, or at least only is mobile, on the fold surfaces of the crystals.

While insufficient evidence exists at this time to determine the origin of the film, it does seem to be polymeric in nature. One puzzling aspect of it, however, is its apparent rigidity. The film is not totally rigid, as evidenced by the observations in which there was seen some pulling of the particles toward the fibrils, but it is rigid enough to have shifted always in the same direction on opposite sides of a given crack. This rigidity persists up to at least $96{ }^{\circ} \mathrm{C}$ in POM, well above $T_{g}$ (while there is some question as to the exact $T_{g}$ for POM, it is well below room temperature [6]).

While further study of this surface film is needed, these preliminary results suggest the presence of an adsorbed polymeric film which could have many ramifications in other areas such as the determination of percent crystallinity or the nature of the molecular folding process. Several of these ramifications are described in the accompanying paper [7]. Further work in these areas may add greatly to our understanding of polymer morphology.

\section{References}

[1] Breedon, J. E., Masters Thesis, Case Western Reserve University (1975).

[2] Geil, P. H., Polymer Single Crystals (Wiley Interscience, New York, 1963).

[3] Frederick, P. S., and Hruska, S. J., J. Appl. Phys. 45, 3215 (1974).

[4] Lauritzen, J. I., Jr. and Hoffman, J. D., J. Appl. Phys. 44, 4340 (1973).

[5] Hoffman, J. D., Davis, G. T., and Lauritzen, J. I., Jr. in Treatise on Solid State Chemistry, N. B. Hannay ed. (Plenum Press, New York, Vol. 3 Chapter 6, 1976).

[6] Sauer, J. A., Richardson, G. C., and Morrow, D. R., J. Macromol. Sci. Revs. Macromol. Chem. C9, 149 (1973).

[7] Hoffman, John D., and Davis, G. Thomas, J. Res. Nat. Bur. Stand. (U.S.), 79A (Phys. and Chem.), No. 5 (Sept.-Oct. 1975).

(Paper 79A5-861) 